\title{
Numerical Simulation of Blood Flow through Asymmetric and Symmetric Occlusion in Carotid Artery
}

\author{
Sahar Ahsaas ${ }^{*}$, Shaligram Tiwari \\ Department of Mechanical Engineering \\ IIT Madras, Chennai-600036, India. \\ sahar.fluid@gmail.com, shaligt@iitm.ac.in
}

\begin{abstract}
Atherosclerosis is hardening and narrowing of arteries due to deposition of fatty materials (plaque) at the inner walls and it is known that haemodynamic plays a major role in the development of such disease. Present work considers non-Newtonian behaviour and pulsatile nature of blood flow in the three-dimensional numerical computations using Ansys Fluent 14.0. Majority of blockages in arteries have been generally reported to be asymmetric and it is observed that it has different flow dynamics than symmetric constrictions. Accordingly, effect of severity (40\% and 60\%) and asymmetry in constriction on possible plaque formation and flow dynamics has been predicted using oscillatory shear index (OSI). The flow field characteristics and OSI variation reveal that an existing occlusion may evolve into multiple occlusions through complex flow behaviour.
\end{abstract}

Keywords: Atherosclerosis, carotid artery, asymmetric occlusion, pulsatile blood flow, non-Newtonian flow of blood.

\section{Introduction}

Atherosclerosis is a form of cardiovascular disease that is commonly due to deposition of LDL-cholesterol, macrophages, smooth muscle cells and calcium in the intima layer of arteries forming plaques. Their formation is predominant in region of vessel branching and bifurcation. Understanding of blood flow hemodynamics has great importance in testing the hypothesis on formation of the cardiovascular disease. The possibility that hemodynamic factors may participate in formation and development of atherosclerosis has encouraged the study of flow through arterial stenosis.

Numerical simulation of arterial stenosis is a non-invasive method of determining the detailed flow pattern associated with the disease. One of the first numerical studies on this type of problem was carried out by Lee and Fung [1]. They analysed the steady flow of blood in a locally constricted tube for Reynolds number (Re) ranging from 0 to 25 . Calculations were not extended to higher Re due to instabilities in the numerical procedure. Deshpande et al. [2] analysed the laminar and steady numerical simulation of blood flow by varying severity of stenose and their results show good agreement with the experiments performed by Young and Tsai [3]. Through their experiments they have given description for measuring pressure loss across the constriction and separation of flow. The nature of flow distal to occlusions, i.e. laminar, transitional or turbulent, was also discussed.

Studies related to above mentioned researches have been performed considering steady flow of blood through arteries. However, the actual blood flow behaviour is pulsatile in nature due to pumping action of heart. For pulsatile flow through stenosis, $\mathrm{Ku}$ et al. [4] carried out experimental study and proposed a correlation between plaque location and wall shear stress, in a way that high shear stress regions are atheroprotective whereas low and oscillating wall shear stress leads to plaque formation. They introduced oscillatory shear index (OSI), a parameter to measure the oscillation in wall shear stress with time interval. Long et al. [5] performed numerical simulations in asymmetric and symmetric constriction in arteries with area reduction of $25 \%, 50 \%$ and $70 \%$. They observed that wall shear stress oscillation in axial direction for asymmetric constriction is larger than symmetric constriction. Tang et al. [6] introduced a 3-D thin wall model with fluidstructure interaction and estimated that asymmetric stenose shows 50\% increase in value of maximum WSS and more negative pressure compared to symmetric stenose. Most of the studies discussed above considered blood as Newtonian fluid, an assumption acceptable for high shear rate flow for example in case of large arteries but it is not valid for low shear regions. The non-Newtonian character of blood is typically due to RBC aggregation, deformation, fibrinogen and lipid loading, etc. Quite a few researchers have presented their work in arterial stenoses by considering non-Newtonian behaviour of blood. Tu et al. [7] have studied two-dimensional steady as well as pulsatile flow and compared three nonNewtonian models, viz. Herschel-Bulkely, Bingham and power law, on the basis of WSS, pressure drop, and velocity. They acclaimed that non-Newtonian models show less distortion of flow pattern distal to stenose. Razavi et al. [8] 
examined the flow dynamics associated in common carotid artery with symmetric stenose, with varying severity from $30 \%$ to $60 \%$, and compared six different non-Newtonian models.

The purpose of present study is to examine the effect of asymmetric constriction in arteries by taking into account the non-Newtonian and pulsatile nature of blood and compared it with symmetric constriction maintaining the constant occlusion level. Comparison and analysis of flow dynamics is performed in two severities of constriction, viz. $40 \%$ and $60 \%$, to observe the effects in different stages of atherosclerosis. Wall shear stress (WSS) and oscillatory shear index (OSI) are the flow parameters that are analysed for the comparison to be made. Velocity plots are presented to understand the flow behaviour that contributes to the difference observed in OSI and WSS in the symmetric and asymmetric constriction.

\section{Methodology}

\subsection{Governing Equations}

In this work, blood is modelled as an incompressible, homogenous and isothermal fluid. The flow through artery is unsteady, laminar, non-Newtonian and fully developed.

To simulate the flow, continuity and Navier-Stokes equations corresponding to conservation of mass and momentum respectively are used.

Continuity equation:

$$
\nabla \cdot \vec{u}=0
$$

Momentum equation:

$$
\frac{\partial(\rho \vec{u})}{\partial t}+\rho(\vec{u} . \nabla) \vec{u}=-\nabla P+\nabla \cdot(\mu \nabla \vec{u})
$$

In the above equations, $\vec{u}, \rho$ and $P$ represent velocity, density and pressure respectively. For defining the rheological properties of blood, dynamic viscosity of fluid $(\mu)$ is described by using non-Newtonian model, i.e. Carreau model, because this model matches well with the experiment data in the entire shear rate region (Cho and Kensey [9]). It gives the constitutive relationship between viscosity and shear rate, defining the shear thinning behaviour of blood. Fig. 1 shows the variation of viscosity with shear rate for the two models and Fig. 2 shows the physiological velocity profile

Carreau Model:

$$
\mu=\mu_{\infty}+\left(\mu_{0}+\mu_{\infty}\right)\left[1+(\lambda \dot{\gamma})^{2}\right]^{(n-1) / 2}
$$

Parameters $\mu_{0}, \mu_{\infty}, \lambda$ and $\dot{\gamma}$ (shear rate) depends on fluid. Zero shear viscosity $\mu_{0}=0.056$ Pa.s, infinite shear viscosity $\mu_{\infty}=0.00345$ Pa.s, power law index $n=0.3568$ and time constant $\lambda=3.3313$ s (Cho and Kensey [9]).

Shear stress is defined as:

$$
\tau=\mu(\dot{\gamma}) \dot{\gamma}
$$

Here $\mu$ is considered to be a function of $\dot{\gamma}$ only. $\dot{\gamma}$ is related to second invariant of rate of deformation tensor $\overline{\bar{D}}$ and is defined as:

$$
\dot{\gamma}=\sqrt{\frac{1}{2} \overline{\bar{D}}: \overline{\bar{D}}}
$$




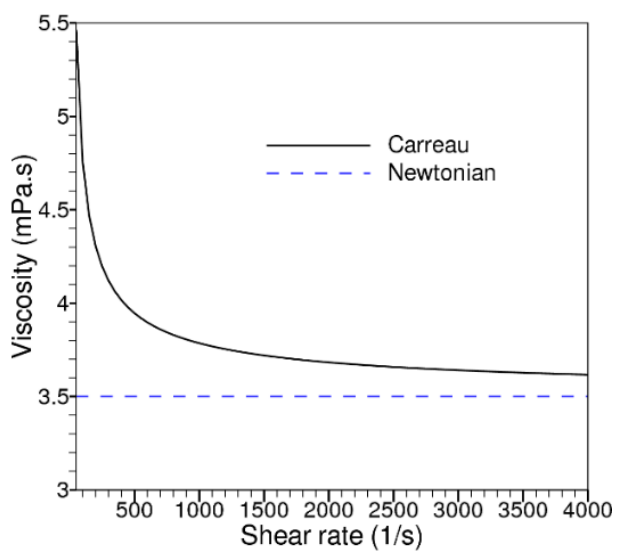

Fig. 1: Viscosity versus shear rate for the two models.

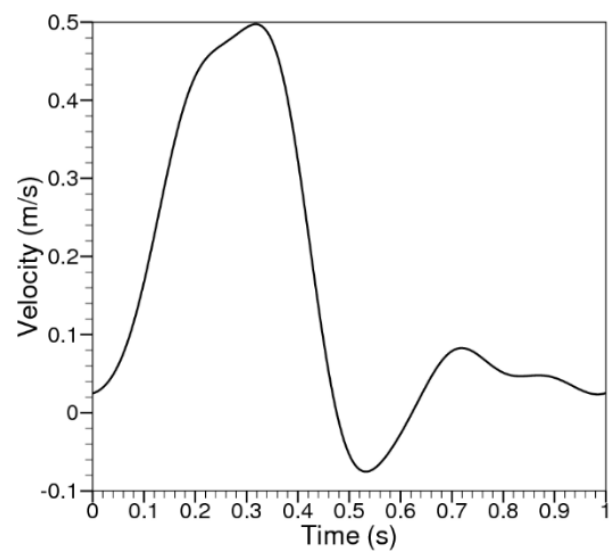

Fig. 2: Physiological velocity profile.

\subsection{Geometry Configuration and Boundary Conditions}

The geometry of stenosis is constructed mathematically by the following bell-shaped Gaussian distribution profile as shown in Fig. 3. Here R refers to radius of the artery while D is the diameter.

Symmetric stenose:

$$
\frac{r(Z)}{R}=1-c e^{-\eta(Z / D)^{2}}
$$

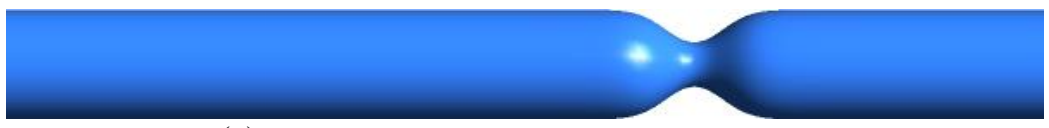

(a)

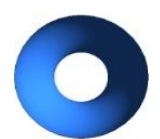

(b)

Asymmetric stenose:

$$
\frac{d(Z)}{D}=1-c e^{-\eta(Z / D)^{2}}
$$

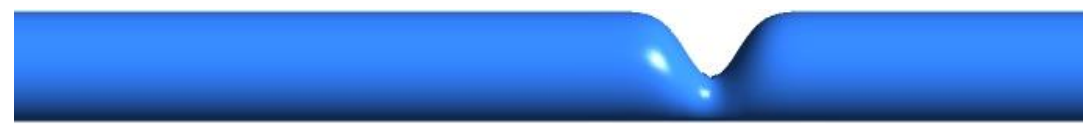

(a)

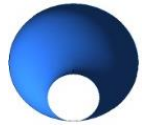

(b)

Fig. 3: Geometry of symmetric and asymmetric stenose (a) side view (b) cross-sectional view.

In this study constriction percentage is defined in terms of $c$ which is equal to $D_{c} / D, D_{c}$ is the constricted diameter measured from throat which is at $\mathrm{Z}=0 \mathrm{~mm}$ and $\mathrm{D}$ is taken as $6.5 \mathrm{~mm}$. The constant $\eta$ which defines the length and shape of stenose region is fixed as 8.0. Upstream and downstream length to throat of stenose is $72.5 \mathrm{~mm}$ and $107.5 \mathrm{~mm}$ respectively.

The boundary conditions imposed on governing equations are as follows:

At wall no-slip boundary conditions is used

$$
u=v=0
$$

At inlet, fully developed velocity profile is given

$$
u(0, r)=2 U\left[1-(r / R)^{2}\right]
$$


$U$ is the physiological pulsatile velocity varying with time shown in Fig.2, the pulse period of this waveform is 1 second and it represents the actual heart rate of a human with heart rate of 60 beats $/ \mathrm{min}$.

At the outlflow section, we assume that the fluid comes out of the domain at its own velocity. It is ensured by imposing zero gauge pressure at outlet.

\subsection{Validation of the Computational Results}

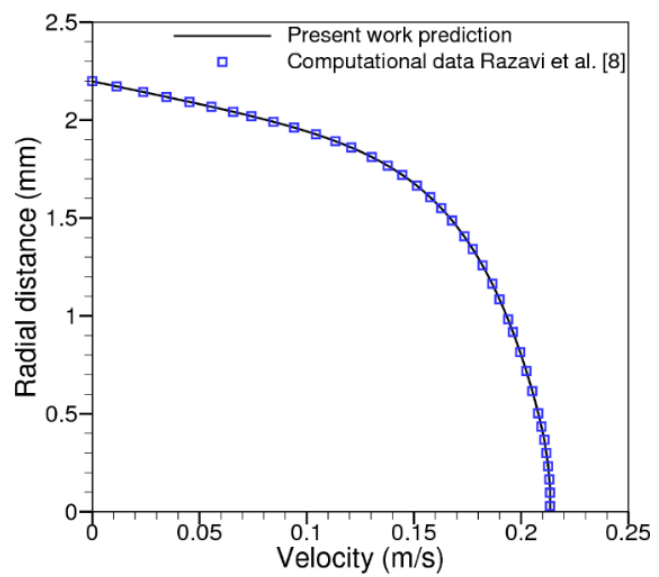

Fig. 4: Comparison of axial velocity at a section $\mathrm{Z}=0 \mathrm{~mm}$ with Razavi et al. [8] computational data.

Computational results are validated by numerical simulation obtained by Razavi et al. [8] for Carreau model in symmetric stenoses. Inlet to domain is physiological waveform of velocity with time period of 0.86 seconds. Results are validated for time $t=0.4$ seconds and $60 \%$ severity, they defined severity in terms of area reduction. Comparison (Fig.4) shows that prediction of present study shows appreciable match with their numerical simulation result.

\subsection{Grid and Numerical Method}

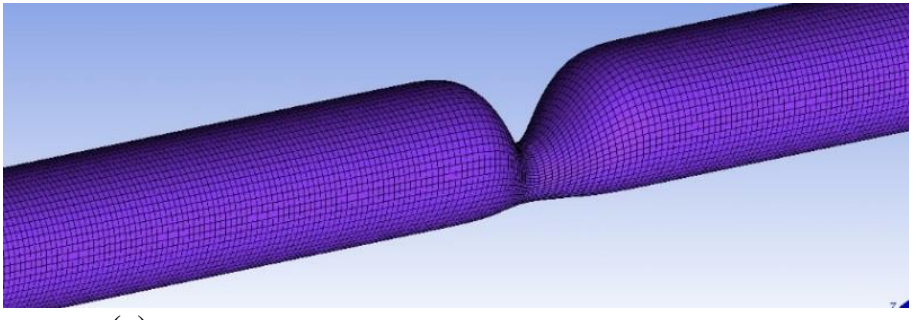

(a)

Fig. 5: (a) Isometric and (b) cross-sectional view of grid mesh.

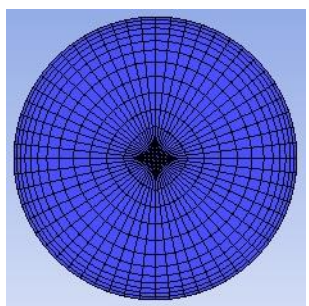

(b)

Structured grid (Fig.5) of hexahedral cells are generated using software ICEM CFD. The grid independence and time independence study has been performed in four refinement and found that 525140 (for symmetric), 619425 (for asymmetric) cells and time step of 0.001 is accurate for this problem. Simulations are performed for three cycles until periodic convergence is achieved. A density of $1050 \mathrm{~kg} / \mathrm{m}^{3}$ is used for blood and target residual of $10^{-6}$ is opted for convergence. The governing equations are solved using software package ANSYS Fluent 14.0 based on finite volume technique and applying SIMPLE scheme.

\section{Results and Discussion}

To understand the flow behaviour around atherosclerosis and its growth and genesis, numerical simulations are performed in asymmetric and symmetric occlusion with two severities $40 \%$ and $60 \%$. Following flow parameters are analysed to observe the effect of flow dynamics on stenosed carotid artery. 


\subsection{Wall shear stress (WSS)}

WSS is one of the important factor in analysis of atherosclerosis in arteries. High WSS can be responsible for rupture of plaque and low and oscillating wall shear stress is known responsible for the formation of plaque. From aspect of maximum wall shear stress in the flow domain Fig.6 shows WSS contour at time $t=0.3$ seconds for $40 \%$ and $60 \%$ severity.
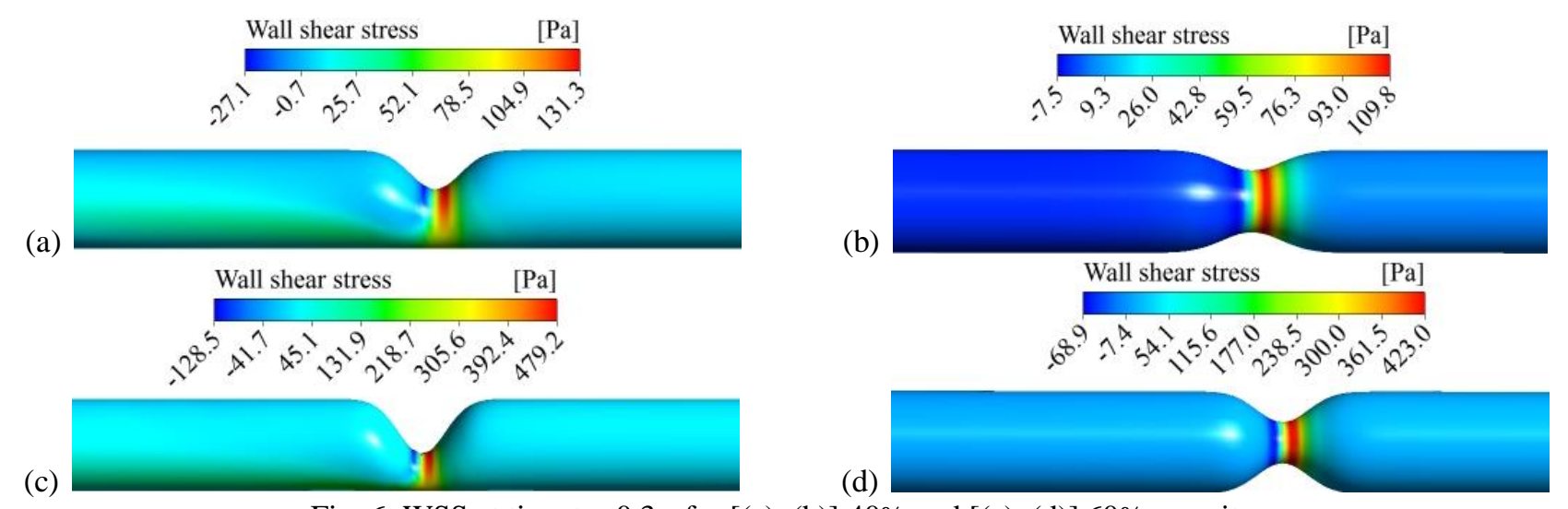

Fig. 6: WSS at time $\mathrm{t}=0.3 \mathrm{~s}$ for $[(\mathrm{a}),(\mathrm{b})] 40 \%$ and $[(\mathrm{c}),(\mathrm{d})] 60 \%$ severity.

Observing the effect of asymmetry (Fig.6 (a) and (c)), WSS distribution is more dispersed compared to symmetric distribution in symmetric stenose (Fig.6 (b) and (d)). WSS value is higher for asymmetric constriction than symmetric, and it is visible from figure that difference increases as severity increases. Asymmetric occlusions are more dangerous from the view of rupture of plaque. Maximum wall shear stress occurs at upstream to throat at about $\mathrm{Z}=-0.2$ to $-0.5 \mathrm{~mm}$ for $\mathrm{t}=0.3$ seconds and for most of the time instant maximum wall shear stress occurs at upstream to throat, so this can lead to possibility of rupture of plaque at this location. There exists an inverse relationship between WSS and formation of plaque, high WSS in the artery makes it atheroprotective. In the case of asymmetric occlusion, velocity profile as shown in Fig.7 (a) is asymmetric about the central axis. Upper wall at stenose region provides more shear $\left(1.21 \times 10^{5} \mathrm{~s}^{-1}\right)$ to fluid element which experiences more local acceleration compared to fluid elements at bottom $\left(7.9 \times 10^{4} \mathrm{~s}^{-1}\right)$ and in case of symmetric stenose (Fig.7 (b)), in which change in velocity and shear experienced by the element at both the walls $\left(1 \times 10^{5} \mathrm{~s}^{-1}\right)$ are similar. As the occlusion level is same it is noted that to maintain the flow rate streamline gets more bend from the upper wall in asymmetric occlusion providing more deformation of fluid elements which on turn provides more wall shear stress to respective wall.

Increase in severity increases the WSS as flow accelerates more due to increase in occlusion level to maintain flow rate. $20 \%$ increase in severity makes the wall shear stress to increase by about 3.7 times of $40 \%$ severity. It is reported in literatures that occlusion of $80 \%$ can lead to turbulence in the flow field due to increase in local Reynolds number near the stenose region.

(a)

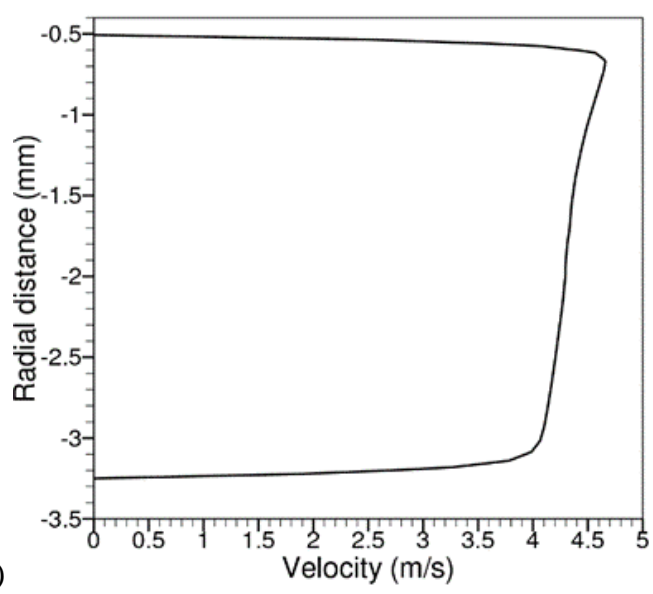

(b)

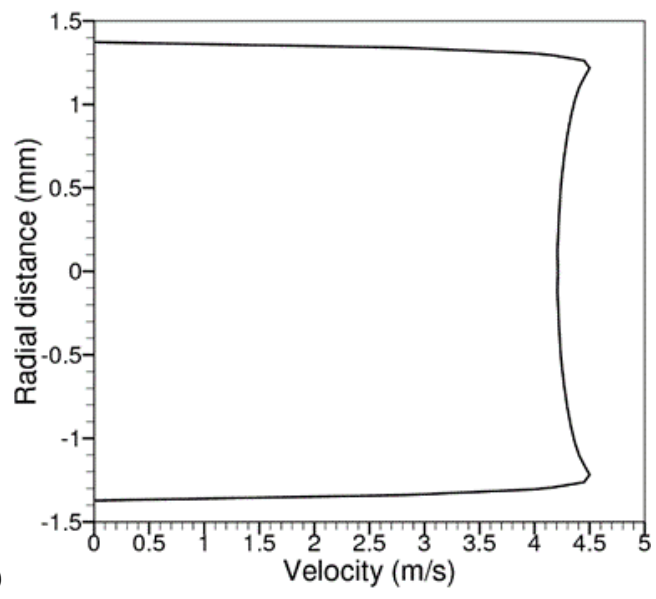

Fig. 7: Velocity profile at $\mathrm{Z}=-0.35 \mathrm{~mm}$ for (a) asymmetric and (b) symmetric constriction at $\mathrm{t}=0.3 \mathrm{~s}$. 


\subsection{Oscillatory shear index (OSI)}

OSI is the mechanical factor related to flow oscillation which monitors the difference between time-averaged WSS magnitude (AWSS) and magnitude of time-averaged WSS vector (AWSSV) values.

$$
\mathrm{AWSS}=\frac{1}{T} \int_{0}^{T}|W \vec{S} S| d t ; \quad \text { AWSSV }=\frac{1}{T}\left|\int_{0}^{T} W \vec{S} S d t\right|
$$

Thus, OSI is calculated as,

$$
\mathrm{OSI}=0.5 \times\left[1-\frac{A W S S V}{A W S S}\right]
$$

Using these values, the OSI defines the WSS vector deflection from blood flow predominant direction, during cardiac cycle. The OSI value can vary from 0.0 for no-cyclic variation to 0.5 for $180^{\circ}$ deflection in WSS direction.

Oscillation in separation and reattachment point corresponds to oscillation in values of WSS vector. OSI distribution for $40 \%$ severity is shown in Fig.8 and Fig.9. Asymmetric stenose has OSI maximum at his upper and bottom wall. As shown by arrow (Fig.8 (a)), location A1 experiences more oscillation in flow field due to the oscillation in reattachment point and it is presented in Fig.8 (b) and (c), flow comes out from contraction like high velocity fluid jet, strikes at bottom wall and moves towards upper wall and the flow gets reattached. Locations at the wall where jet strikes changes with time intervals and this creates an oscillation of flow parameters at that locations.

(a)

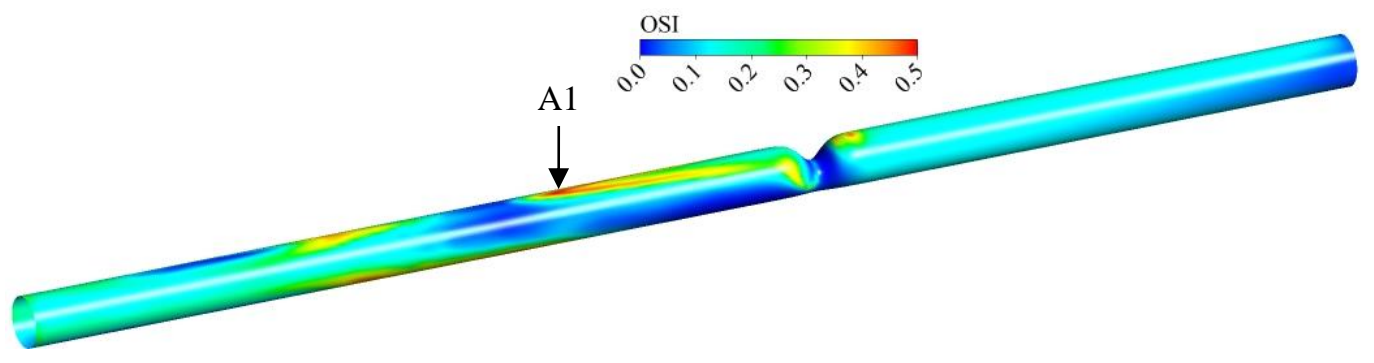

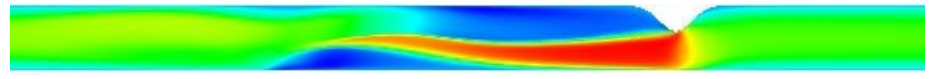

(b)

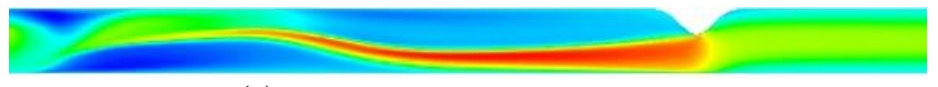

(c)

Fig. 8: OSI for (a) asymmetric occlusion with $40 \%$ severity; corresponding velocity contours at (b) $t=0.2 \mathrm{~s}$ (a) and (c) $t=0.4 \mathrm{~s}$.

OSI distribution in symmetric stenose is shown in Fig.9. Maximum OSI is observed at $\mathrm{Z}=67 \mathrm{~mm}$ (A2 in Fig.9). To understand the reason for high OSI values at a region $Z=55 \mathrm{~mm}$ to $80 \mathrm{~mm}$, WSS values are presented in Table 1 at different locations from throat for different time intervals, as it is difficult to analyse and explain the reason from velocity plots. Summation of WSS vector has least value at location $\mathrm{Z}=67 \mathrm{~mm}$ (Table 1), so this gives a confirmation of oscillation of WSS about its mean giving rise to OSI at that particular region. For much closer approximation of WSS oscillation, more number of time intervals would give better accuracy.

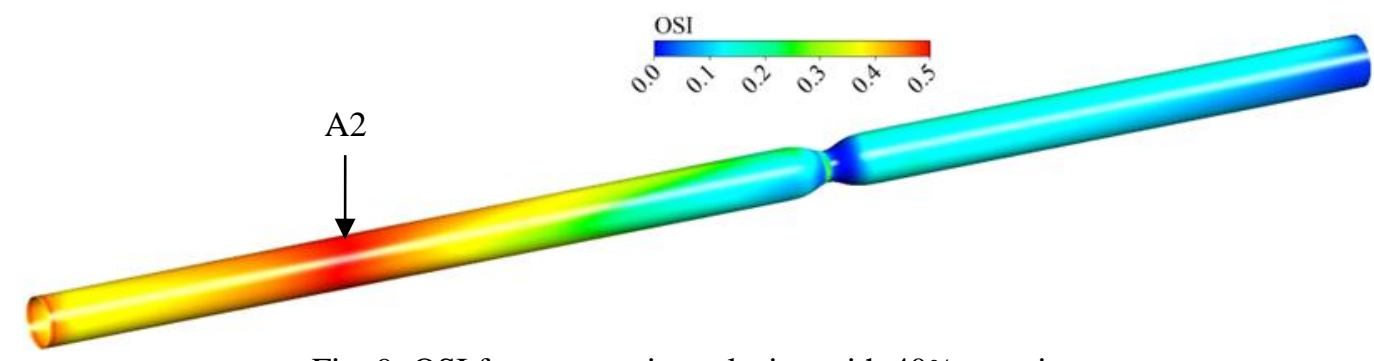

Fig. 9: OSI for symmetric occlusion with $40 \%$ severity. 
Table 1: WSS values at different locations for different time intervals.

\begin{tabular}{|c|c|c|c|c|c|c|c|c|c|c|}
\hline \multirow{2}{*}{$\begin{array}{c}\text { Time } \\
\text { (seconds) }\end{array}$} & \multicolumn{10}{|c|}{ Distance from throat $(\mathbf{Z})$ in $\mathbf{~ m m}$} \\
\cline { 2 - 11 } & $\mathbf{1 0}$ & $\mathbf{2 0}$ & $\mathbf{3 0}$ & $\mathbf{4 0}$ & $\mathbf{5 0}$ & $\mathbf{6 7}$ & $\mathbf{7 0}$ & $\mathbf{8 0}$ & $\mathbf{9 0}$ & $\mathbf{1 0 0}$ \\
\hline $\mathbf{0 . 1}$ & -0.168 & 2.516 & 2.516 & 2.5 & 2.5 & 2.5 & 2.5 & 2.5 & 2.5 & 2.51 \\
\hline $\mathbf{0 . 2}$ & -3.42 & -3.761 & -3.0447 & -1.46 & 1.2 & 4.09 & 4.173 & 4.26 & 4.26 & 4.345 \\
\hline $\mathbf{0 . 3}$ & -3.324 & -3.588 & -3.25 & -2.48 & -1.75 & -0.43 & -0.2432 & 0.325 & 0.8 & 1.2 \\
\hline $\mathbf{0 . 4}$ & -0.908 & -1.894 & -1.9 & -2.68 & -3.2 & -3.4 & -3.356 & -3.16 & -2.9 & -2.55 \\
\hline $\mathbf{0 . 5}$ & -0.027 & -0.5702 & -1.292 & -2 & -2.48 & -3 & -3.0369 & -3.15 & -3.25 & -3.25 \\
\hline $\mathbf{0 . 6}$ & 0.6875 & 0.4631 & 0.21347 & 0.0235 & -0.126 & -0.274 & -0.3265 & -0.36 & -0.38 & -0.422 \\
\hline $\mathbf{0 . 7}$ & 1.38 & 1.2008 & 1.108 & 1.008 & 0.95 & 0.842 & 0.83112 & 0.81 & 0.8 & 0.7965 \\
\hline $\mathbf{0 . 8}$ & -0.247 & 0.362 & 0.32689 & 0.285 & 0.235 & 0.18 & 0.16 & 0.154 & 0.14 & 0.137 \\
\hline $\mathbf{0 . 9}$ & -0.111 & 0.0415 & 0.00766 & 0.23 & 0.215 & 0.184 & 0.178 & 0.165 & 0.158 & 0.155 \\
\hline $\mathbf{1}$ & -0.014 & 0.0025 & 0.049 & 0.047 & 0.109 & 0.104 & 0.10554 & 0.1 & 0.095 & 0.0927 \\
\hline$\sum W \vec{S} S \mid=$ & $\mathbf{6 . 1 5 1 5}$ & $\mathbf{5 . 2 2 7 3}$ & $\mathbf{5 . 2 6 5}$ & $\mathbf{4 . 5 2 8}$ & $\mathbf{2 . 3 4 7}$ & $\mathbf{0 . 7 9 6}$ & $\mathbf{0 . 9 8 5}$ & $\mathbf{1 . 7}$ & $\mathbf{2 . 2 2 3}$ & $\mathbf{3 . 0 1 4 2}$ \\
\hline
\end{tabular}

OSI distribution at $60 \%$ severe artery is shown in Fig. 10. In case of asymmetric stenose (Fig. 10 (a)) OSI values are higher at side walls much different from symmetric stenose (Fig. 10 (b)). Observing the effect of severity, at higher severity there is large distribution of high OSI values downstream to stenose compared to $40 \%$ severe stenose. Monitoring the flow behaviour in two severities shows that at $60 \%$ severe symmetric occlusion, flow parameters are not symmetrical and shows small deviation.

(a)

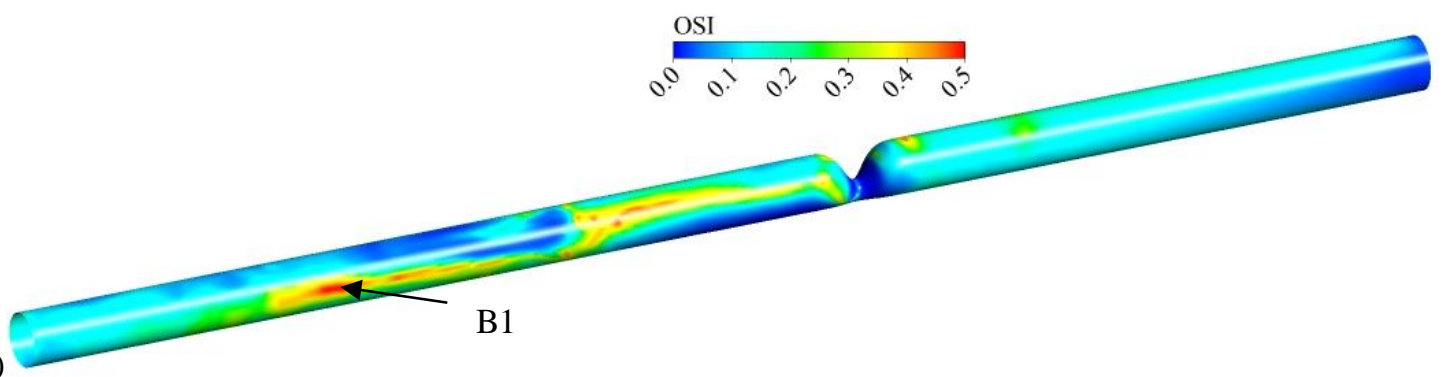

(b)

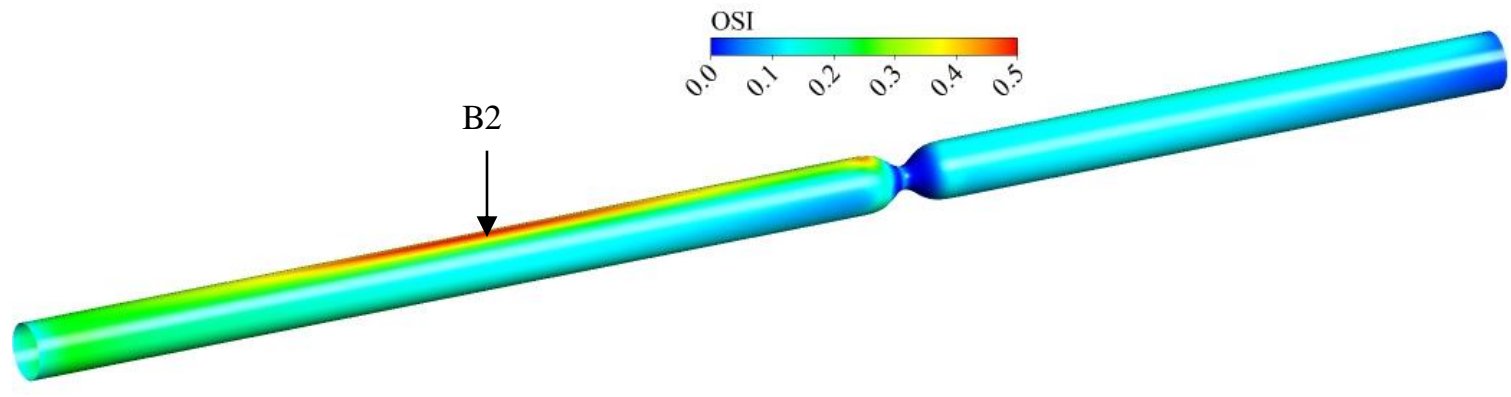

Fig. 10: OSI for (a) asymmetric and (b) symmetric occlusion for $60 \%$ severity.

Observing Fig.11 and Fig.12 it is visible that change in flow parameters are occurring at certain region with time. For asymmetric stenose much change in the flow parameters are observed at upper side wall (Fig. 11). This changes are dominant at locations $Z=31.0 \mathrm{~mm}$ and $Z=64.25 \mathrm{~mm}$ (B1 in Fig. 10 (a)). For symmetric stenose (Fig.12), upper and lower wall have changes dominant at locations $Z=38.5 \mathrm{~mm}, Z=51.3 \mathrm{~mm}$ (B2 in Fig. 10 (b)) and $Z=63.3 \mathrm{~mm}$. The changes in the flow parameters at these locations lead to high OSI shown in Fig 10. 


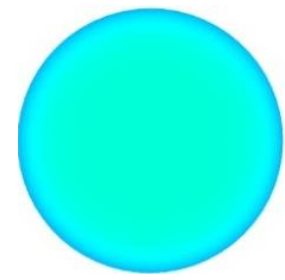

(a)

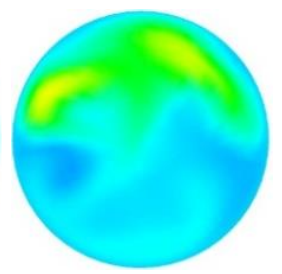

(b)

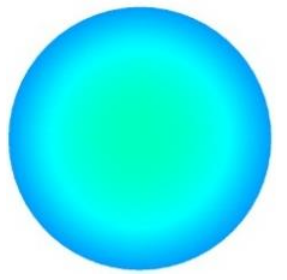

(c)

Fig. 11: Cross- section $(Z=64.25 \mathrm{~mm})$ of velocity contours for asymmetric constriction at time (a) $\mathrm{t}=0.2 \mathrm{~s}$, (b) $0.4 \mathrm{~s}$ and (c) $0.9 \mathrm{~s}$.

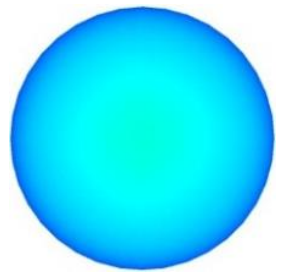

(a)

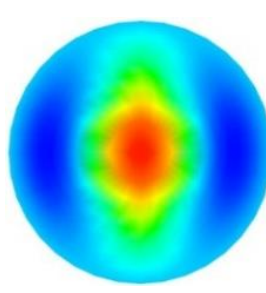

(b)

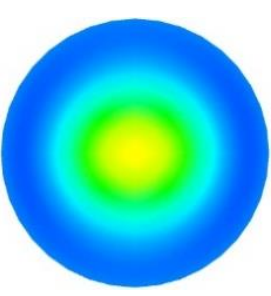

(c)
Fig. 12: Cross-section $(Z=51.32 \mathrm{~mm})$ of velocity contours for symmetric constriction at time (a) $\mathrm{t}=0.2 \mathrm{~s}$, (b) $0.4 \mathrm{~s}$ and (c) $0.9 \mathrm{~s}$.

\section{Conclusion}

The study presented the comparison between symmetric and asymmetric occlusion in two severities $40 \%$ and $60 \%$ severity by analysing WSS distribution and OSI. Analysis shows that distribution of WSS and OSI is significantly different in two constrictions and as well as in two severities. Thus performing numerical simulation in occluded arteries assumption of complete symmetry of occlusion can lead to significant errors. Analysing the effect of severity in symmetric occlusion it is noted that at higher severity (60\%) flow becomes non-symmetric. OSI distribution is predominant at downstream region of occlusion, so there can be possibility of developing plaque at this locations, disturbance in flow dynamics from already developed occlusion can lead to multiple occlusions in the arteries.

\section{References}

[1] J. S. Lee and Y. F. Fung, "Flow in locally constricted tubes at low Reynolds number," J. of Applied Mechanics., vol. 37, pp. 9-16, 1970.

[2] M. D. Deshpande, D. P. Giddens, and R. F. Mabon, "Steady laminar flow through modelled vascular stenosis," J. of Biomechanics., vol. 9, pp. 165-174, 1976.

[3] D. F. Young and F. Y. Tsai, "Flow characteristics in models of arterial stenoses-I. Steady flow," J. of Biomechanics., vol.6, pp. 395-410, 1973.

[4] D. N. Ku, D. P. Giddens, C. K. Zarins, and S. Glagov, "Pulsatile flow and atherosclerosis in the human carotid bifurcation," J. of Arteriosclerosis., vol. 5, no. 4, pp. 293-302, 1985.

[5] Q. Long, X. Y. Xu, K. V. Ramnarine, and P. Hoskins, "Numerical investigation of physiologically realistic pulsatile flow through arterial stenose," J. of Biomechanics., vol. 34, pp. 1229-1242, 2001.

[6] D. Tang, C. Yang, and D. N. Ku, "A 3-D thin wall model with fluid-structure interactions for blood flow in carotid arteries with symmetric and asymmetric stenosis," Computer and Structures, vol. 72, pp. 357-377, 1999.

[7] C. Tu and M. Deville, "Pulsatile flow of non-Newtonian fluids through arterial stenosis," J. of Biomechanics., vol. 29, pp. 899-908, 1996.

[8] A. Razavi, E. Shirani, and M. R. Sadeghi, "Numerical solution of blood pulsatile flow in a stenosed carotid artery using different rheological model," J. of Biomechanics., vol. 44, pp. 2021-2030, 2011.

[9] Y. I. Cho and K.R. Kensey, "Effects of the non-Newtonian viscosity of blood on hemodynamics of diseased arterial flows: part 1, steady flows," Biorheology., vol. 28, pp. 241-262, 1991. 